\title{
Influência do rio Negro sobre o status nutricional de juvenis de curimatã Prochilodus nigricans (Characiformes; Prochilodontidae) no médio rio Solimões-Amazonas, Brasil
}

\author{
Carlos Eduardo MOUNIC-SILVA ${ }^{1}$, Rosseval Galdino LEITE ${ }^{2}$
}

RESUMO

As diferenças limnológicas entre as águas pretas do rio Negro e as águas brancas do rio Solimôes-Amazonas podem ter influência sobre o status nutricional de peixes detritívoros pela diferença nas fontes dos recursos alimentares entre estes sistemas. Para verificar a influência do rio Negro sobre o status nutricional de juvenis de curimatá Prochilodus nigricans foi medida a condutividade em pontos de amostragem e estimado o fator de condição (K, a partir da relação peso/comprimento) dos juvenis em três áreas de inundação do médio rio Solimôes-Amazonas: (1) com influência do rio Negro, próxima a Manaus; (2) sem influência do rio Negro, próxima a Manaus e (3) sem influência do rio Negro, em um trecho do Solimóes-Amazonas 500 $\mathrm{km}$ a montante de Manaus. A condutividade nos pontos de amostragem com influência do rio Negro foi significativamente menor do que as outras áreas e o $\mathrm{K}$ dos juvenis de águas com influência do rio Negro foi significativamente maior. A alta seletividade do curimatã pode dificultar a assimilação de itens alimentares quando estes estão em alta abundância. Em adição, as águas pretas possuem detritos com alto potencial de assimilação devido à sua qualidade protéica, o que demonstra a grande importância das florestas inundadas por água pretas para os detritívoros amazônicos.

PALAVRAS-CHAVE: condutividade; fator de condição; pesca; detritívoros; bacia amazônica.

\section{Influence of river Negro on nutritional status of curimatã Prochilodus nigricans (Characiformes; Prochilodontidae) juveniles in the middle river Solimões-Amazonas, Brazil}

\begin{abstract}
The limnological differences between the black waters of the Negro river and the white waters of the Solimóes-Amazonas river can influence the nutritional status of detritivorous fishes by the difference in the food sources between these systems. To check the influence of the Negro river on the nutritional status of curimatá juveniles Prochilodus nigricans we estimated the conductivity in the sampling points and condition factor ( $\mathrm{K}$, from weight/length relationship) of the juveniles in three flooding areas of the middle Solimóes-Amazon river: (1) with Negro river influence, near Manaus (2) without Negro river influence, near Manaus and (3) without Negro river influence, in a stretch of the Solimóes-Amazonas $500 \mathrm{~km}$ upstream from Manaus. The conductivity in the sampling points with Negro river influence was significantly lower than other areas and the $\mathrm{K}$ of juveniles influenced by waters of the Negro river was significantly higher than two other areas. The high selectivity of curimatá may limit the assimilation of food items when they are in high abundance. In addition, the black water detritus have high potential for assimilation because of its protein quality, which demonstrates the importance of flooded forests by black waters for Amazonian detritivorous.
\end{abstract}

KEYWORDS: conductivity; condition factor; fishery; detritivorous; Amazon basin.

1 Instituto Federal de Educação, Ciência e Tecnologia de Rondônia (IFR0)/Campus Ariquemes - Rodovia R0-257, Km 13, Ariquemes/R0 - CEP $76870-970$ E-mail: carlos.silva@ifro.edu.br

2 Instituto Nacional de Pesquisas da Amazônia (INPA) - Coordenação de Pesquisas em Biologia Aquática (CPBA) - Av. André Araújo, 2936 , Aleixo, Manaus/AM - CEP 69060-001. E-mail: rosseval@gmail.com 


\section{INTRODUÇÃO}

No médio rio Solimóes-Amazonas, nas proximidades da cidade de Manaus, dois tipos de águas inundam sazonalmente as planícies fluviais da região: as águas brancas do rio SolimõesAmazonas e as águas pretas do rio Negro. Estes dois tipos de águas apresentam acentuadas diferenças, especialmente a condutividade, cujas medidas contrastam marcadamente entre águas brancas e pretas (Sioli 1984). Esta diferença está diretamente associada às origens e padrôes hidrogeológicos entre as bacias hidrográficas de rios de águas pretas e brancas. O rio Negro, por exemplo, é originário do interior da bacia amazônica e caracterizado pela baixa concentraçấo de íons dissolvidos, ao contrário do Solimôes-Amazonas que carreia altas concentrações de íons dissolvidos, os quais se originam da erosão pluvial na cordilheira andina. Enquanto no rio Solimôes-Amazonas, a condutividade é muito elevada, com uma quantidade de íons solubilizados podendo chegar a $150 \mu \mathrm{S}$ ou mais e, um valor médio de $80 \mu \mathrm{S}$, no rio Negro a condutividade alcança valores máximos de $25 \mu \mathrm{S}$ e média em torno de $10 \mu S$ (Sioli 1984).

Adicionalmente, as diferenças de ordem química não são as únicas características limnológicas que distinguem estes dois sistemas. As fontes dos recursos alimentares entre os rios de águas brancas e os rios de águas pretas têm origens diferenciadas. Em virtude da baixa concentração de nutrientes, os rios de águas pretas possuem uma produção primária in situ mais pobre quando comparada aos sistemas de águas brancas (Barthem e Goulding 2007). Goulding et al. (1988) sugerem que os detritos sáo muito importantes em sistemas pobres em nutrientes, tais como áreas de inundação de rios de águas pretas ("igapó"), sendo que o material alóctone tem papel fundamental na constituição das cadeias alimentares detritívoras, preponderantes nestes ecossistemas.

O curimatã Prochilodus nigricans (Prochilodontidae) é uma espécie detritívora da bacia amazônica, que se alimenta de algas perifíticas, de microrganismos e da matéria orgânica em decomposição, geralmente depositada no fundo de rios e lagos de planície de inundação (Santos et al. 2006). Ocupa tanto rios de águas pretas e claras como também rios de águas brancas, apresenta desova total no início da enchente, quando há a formação de grandes cardumes que migram em direção aos rios de águas brancas para desovar (Santos et al. 2006; Lima e Araújo-Lima 2004). As larvas derivam rio abaixo penetrando em áreas alagáveis no início das inundaçôes por meio de canais, paranás e igarapés, onde encontram alimento e abrigo para seu crescimento até a fase juvenil (Araújo-Lima e Oliveira 1998; Sanchéz-Botero e Araújo-Lima 2001). O curimatã tem grande importância nas pescarias comerciais e artesanais da Amazônia Central e ocupou o terceiro lugar em desembarques pesqueiros da cidade de Manaus entre os anos de 1976 e 2003, o que corresponde a $12 \%$ do total desembarcado neste período
(Barthem e Goulding 2007). Entre 1994 e 1996, esteve em segundo lugar no total desembarcado em Manaus, perdendo somente para o jaraqui, o qual apresenta duas espécies agrupadas em um único recurso pesqueiro (Semaprochilodus insigins e S. taeniurus) (Batista e Petrere Jr. 2003).

Nas populações de peixes detritívoros da região do encontro das águas dos rios Solimôes-Amazonas e Negro, a condiçấo nutricional de peixes em fases iniciais de vida pode ser afetada em funçáo das diferenças na base das cadeias tróficas entre as águas brancas e pretas. Este fato exige uma atenção especial dos tomadores de decisão no campo do gerenciamento pesqueiro, pois algumas áreas de planície de inundação desta regiáo podem aumentar o sucesso de recrutamento de populaçóes pesqueiras (Forsberg et al. 1993; Benedito-Cecílio et al. 2000).

Portanto, pela importância do curimatá para a pesca na Amazônia Central, e em virtude das diferenças na base da estruturaçáo trófica entre sistemas de águas pretas e brancas e suas implicaçóes para o estado nutricional de juvenis de espécies detritívoras amazônicas, este trabalho teve como objetivo verificar a influência de águas pretas sobre o estado nutricional de juvenis de curimatã em áreas de inundação do encontro das águas dos rios Solimôes-Amazonas e Negro.

\section{MATERIAL E MÉTODOS}

\section{Área de estudo}

Os juvenis de curimatã foram capturados em três áreas de planície de inundação do eixo Solimóes-Amazonas, duas delas na junçáo do rio Negro com o rio Solimôes-Amazonas (encontro das águas); uma com influência de águas pretas (área 1) e outra sem influência (área 2); e a terceira área, no médio Solimóes-Amazonas, a uma distância de $500 \mathrm{~km} \mathrm{a}$ montante de Manaus (área 3), sem influência de águas pretas, nas proximidades do município de Coari (AM) (Figura 1).

\section{Amostragem, processamento e análise de dados}

As capturas foram realizadas mensalmente durante o período de enchente do rio Solimóes-Amazonas e também recria de juvenis de $P$. nigricans, de dezembro de 2007 a maio de 2008 , com um total de 220 pontos amostrados nas três áreas de estudo. Em cada ponto, as capturas dos juvenis de curimatá foram realizadas com dois aparelhos de pesca, a rede-de-cerco ("redinha") e a rede-de-mão ("puçá"), sendo explorados principalmente habitats marginais com vegetação aquática em áreas de planície de inundação e às margens do rio Solimôes-Amazonas.

Os pontos de captura foram caracterizados pela condutividade com o objetivo de verificar a influência de águas pretas sobre o respectivo ponto de captura. Utilizando este critério, foram feitos 156 registros de condutividade, 


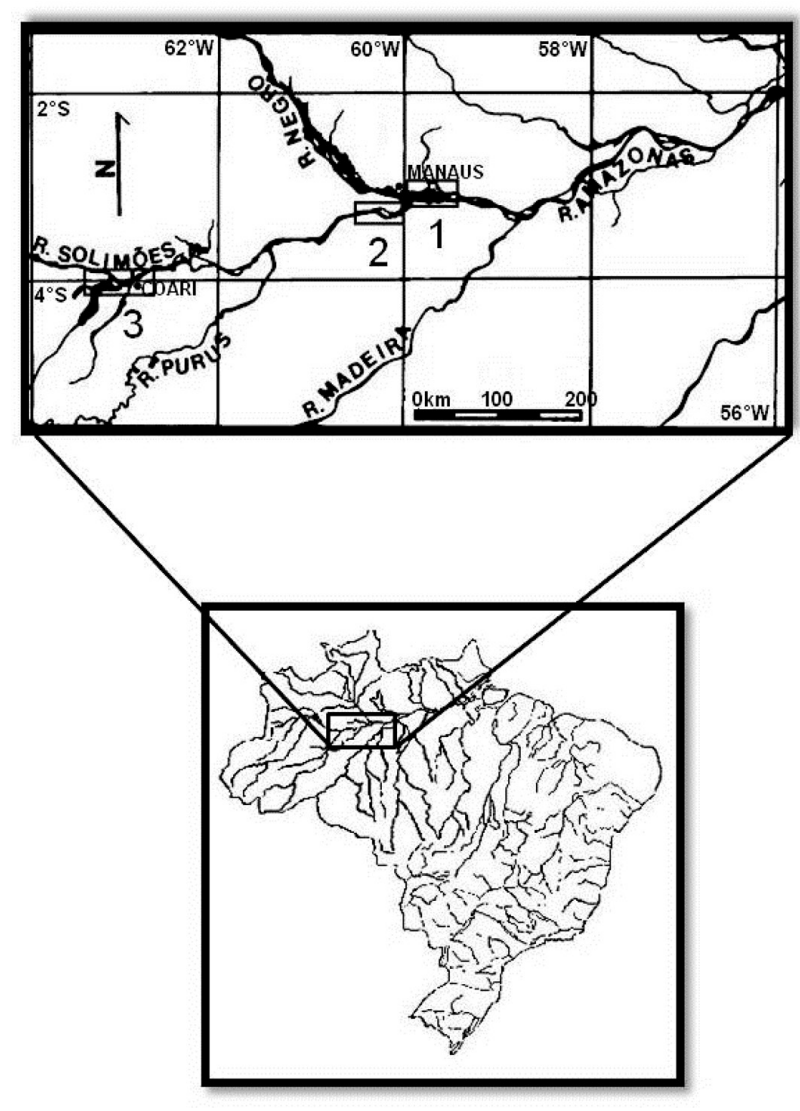

Figura 1 - Localização das três áreas de captura (indicadas no mapa pelos números 1,2 e 3) dos juvenis de curimatã Prochilodus nigricans na Amazônia Central (Brasil).

distribuídos uniformemente para cada uma das três áreas de estudo, ou seja, 52 registros para cada área. Os dados de condutividade foram analisados por meio de uma ANOVA de Kruskal-Wallis $(\mathrm{p}<0,05)$ tendo em vista a forte heterocedasticidade dos dados (Zar 1996), com três grupos sendo comparados: área 1, área 2 e área 3. Uma análise $a$ posteriori de Dunn para múltiplas comparaçóes foi utilizada a um nível de significância menor do que 5\% (p<0,05).

Do total dos 1561 indivíduos capturados, três subamostras, correspondentes a cada área estudada, foram extraídas ao acaso para homogeneizaçáo dos tamanhos de amostra entre áreas, sendo selecionados indivíduos com comprimento total acima de $10 \mathrm{~mm}$ e abaixo de $40 \mathrm{~mm}$, utilizando ictiomêtro digital para o comprimento e balança digital para o peso. Desta forma, na área 1 (com influência de águas pretas, próxima a Manaus) foram analisados 220 indivíduos, na área 2 (sem influência de águas pretas, próxima a Manaus) foram analisados $218 \mathrm{e}$ na área 3 (sem influência de águas pretas, afastada $500 \mathrm{~km}$ de Manaus) 210 indivíduos, totalizando 648 indivíduos analisados.
O fator de condição $(\mathrm{K})$ estimado a partir da relação peso/ comprimento (Le Cren 1951) foi utilizado para verificar a condição nutricional dos peixes em cada uma das áreas. Para a obtenção do fator de condição foi utilizada a fórmula (Vazzoler 1996):

$$
\mathrm{K}=\mathrm{Wt} / \mathrm{Lt}^{\theta} \text {. }
$$

$\mathrm{Na}$ qual, Wt denota o peso total (g); Lt representa o comprimento total $(\mathrm{cm})$ e $\theta$ corresponde ao índice de alometria, retirado da relação potencial entre peso/ comprimento. As diferenças no fator de condição entre as áreas 1, 2 e 3 foram verificadas da mesma maneira que as diferenças na condutividade devido à heterocedasticidade dos dados de fator de condição. Desta forma, uma ANOVA de Kruskal-Wallis $(\mathrm{p}<0,05)$ também foi aplicada para este caso (Zar 1996), com teste de Dunn para comparaçóes múltiplas $(\mathrm{p}<0,05)$ a posteriori.

\section{RESULTADOS}

Os peixes estudados apresentaram médias de peso e comprimento muito próximas entre áreas no sentido dos valores obtidos (Tabela 1), fato que torna as comparaçóes mais robustas em virtude da semelhança no comportamento alimentar entre os juvenis das três áreas estudadas.

As medidas de peso e comprimento dos três grupos de peixes estudados deram origem às seguintes relaçóes peso/comprimento: área 1: $\operatorname{Ln} W t=\operatorname{Ln}(-14,81)+3,34 \operatorname{Ln} L t$; área 2: $\operatorname{Ln} W t=\operatorname{Ln}(-15,54)+3,48 \operatorname{Ln} L t$ e área 3: $\operatorname{Ln} W t=\operatorname{Ln}(-$ $15,21)+3,41 \mathrm{Ln} L t$.

A área 1 (com influência do rio Negro) apresentou a menor média de condutividade dos pontos de amostragem (Tabela 2), com diferença estatisticamente significativa em relação às áreas 2 e 3 (sem influência do rio Negro), dada pela análise de Kruskal-Wallis $(\mathrm{H}=17,932, \mathrm{~N}=156, \mathrm{GL}=2)$.

Tabela 1 - Médias e desvios-padrão de peso e comprimento em juvenis de curimatã Prochilodus nigricans nas três áreas de estudo no médio rio Solimões-Amazonas.

\begin{tabular}{ccc}
\hline Área & Peso $(\mathrm{g})$ & Comprimento $(\mathrm{mm})$ \\
\hline 1 & $0,361( \pm 0,315)$ & $22,90( \pm 6,07)$ \\
2 & $0,358( \pm 0,276)$ & $22,92( \pm 5,66)$ \\
3 & $0,388( \pm 0,336)$ & $23,07( \pm 6,75)$ \\
\hline
\end{tabular}

Tabela 2 - Médias e desvios-padrão obtidos para a condutividade e fator de condição (K) de juvenis de curimatã Prochilodus nigricans nas três áreas de estudo no médio rio Solimões-Amazonas.

\begin{tabular}{ccc}
\hline Área & Condutividade $(\mu \mathrm{S})$ & Fator de Condição $(\mathrm{K})$ \\
\hline 1 & $65,48 \pm 40,01$ & $0,0177 \pm 0,0018$ \\
2 & $91,83 \pm 24,84$ & $0,0163 \pm 0,0015$ \\
3 & $81,53 \pm 30,27$ & $0,0163 \pm 0,0022$ \\
\hline
\end{tabular}


Estas duas últimas apresentaram condutividades similares, não apresentando diferenças estatísticas significativas, conforme teste de múltiplas comparaçóes a posteriori (Tabela 3).

A análise de Kruskal-Wallis e teste de múltiplas comparaçóes para o $\mathrm{K}$ mostraram diferenças estatísticas entre as médias de $\mathrm{K}$ dos juvenis capturados na área 1 e a média do $\mathrm{K}$ dos juvenis das áreas 2 e $3(\mathrm{H}=85,061, \mathrm{~N}=649, \mathrm{GL}=2)$, sendo estas duas últimas não diferentes estatisticamente (Tabela 3).

Tabela 3 - Matriz de comparações múltiplas (teste de Dunn) para diferenças na média de condutividade $(\mu \mathrm{S})$ dos pontos de amostragem e fator de condição (K) dos juvenis de curimatã Prochilodus nigricans entre as três áreas de estudo no médio rio Solimões-Amazonas.

\begin{tabular}{cccccccc}
\hline \multirow{2}{*}{ Área } & \multicolumn{3}{c}{1} & \multicolumn{2}{c}{2} & \multicolumn{2}{c}{3} \\
\cline { 2 - 8 } & & $\mu S$ & $K$ & $\mu S$ & $K$ & $\mu S$ & $K$ \\
\hline \multirow{2}{*}{1} & $\mu S$ & - & - & $\mathbf{p}<\mathbf{0 , 0 5}$ & - & $\mathbf{p}<\mathbf{0 , 0 5}$ & - \\
& $K$ & - & - & - & $\mathbf{p}<\mathbf{0 , 0 5}$ & - & $\mathbf{p}<\mathbf{0 , 0 5}$ \\
\hline \multirow{2}{*}{2} & $\mu S$ & $\mathbf{p}<\mathbf{0 , 0 5}$ & - & - & - & $p>0,05$ & - \\
& $K$ & - & $\mathbf{p}<\mathbf{0 , 0 5}$ & - & - & - & $\mathbf{p}>0,05$ \\
\hline \multirow{2}{*}{3} & $\mu S$ & $\mathbf{p}<\mathbf{0 , 0 5}$ & - & $p>0,05$ & - & - & - \\
& $K$ & - & $\mathbf{p}<\mathbf{0 , 0 5}$ & - & $\mathbf{p}>0,05$ & - & - \\
\hline
\end{tabular}

Os resultados com $p$ significativo estão em negrito.

\section{DISCUSSÃO}

$\mathrm{Na}$ região do encontro das águas dos rios Negro e Solimóes-Amazonas, formando o que se considera o rio Amazonas (Barthem e Goulding 2007), a margem esquerda deste rio é marcadamente uma extensão do rio Negro, com as áreas de inundação desta margem alagadas pelas águas pretas do rio Negro, criando extensas áreas de "igapó” (florestas inundadas por águas pretas). A área 1, com influência do rio Negro, apresentou pontos de amostragem dentro destas áreas, o que explica seus menores valores de condutividade em relação as áreas 2 e 3. Estas duas últimas não apresentaram diferenças significativas de condutividade, uma vez que seus pontos de amostragem estavam sob pouca ou nenhuma influência de águas pretas.

Com relação à produtividade biológica, as áreas alagadas por águas brancas ou barrentas, chamadas áreas de várzea, apresentam uma alta produção primária e, consequentemente, grande abundância de microinvertebrados (Leite e AraújoLima 2002). Isto poderia gerar uma maior disponibilidade de recursos alimentares e, desta forma, um maior fator de condição para os juvenis de curimatá coletados nas áreas 2 e 3 (rio Solimóes-Amazonas) do que para os da área 1 (com influência do rio Negro).

No entanto, a maior quantidade de nutrientes dos sistemas de águas brancas comparado aos sistemas de águas pretas (Sioli 1984), que possibilitam uma maior disponibilidade de recursos e estruturação de habitats tipo "berçário" (Sanchéz-
Botero e Araújo-Lima 2001; Petry et al. 2003), pode não ser a única condição importante para o desenvolvimento inicial e sucesso de recrutamento às fases posteriores de vida em $P$. nigricans. Os juvenis de curimatã coletados em áreas com influência de águas pretas apresentaram maior média do fator de condiçáo o que pode indicar o alto grau de adaptação desta espécie aos sistemas aquáticos desta natureza.

A diferença nas fontes de energia para detritívoros amazônicos entre ambientes eutróficos, tais como as áreas de inundação do Solimôes-Amazonas, e ambientes oligotróficos, tais como os "igapós" do rio Negro, foi verificada por Benedito-Cecílio e Araújo-Lima (2002). Estes autores demonstraram que para adultos do jaraqui escama-grossa S. insignis, um detritívoro de grande importância comercial na Amazônia Central, as fontes de energia em ambientes oligotróficos foram baseadas principalmente em plantas $\mathrm{C}_{3}$, incluindo plantas vasculares, detritos e algas (principalmente perifíton) e, em ambientes eutróficos, estas fontes eram em grande parte plantas $\mathrm{C}_{4}$, como os capins aquáticos.

Adicionalmente, Leite et al. (2002) e Silva-Soares (2010) verificaram que em larvas e juvenis de $S$. insignis, medindo entre 5 e $17 \mathrm{~mm}$ no primeiro estudo e, entre 16 e $60 \mathrm{~mm}$ no segundo, houve grande participação dos detritos (plantas, $\mathrm{C}_{3}$ ) na dieta desta espécie, o que pode dar fortes indícios da importante participação dos detritos também na alimentação inicial de outros detritívoros, tais como P. nigricans.

Gomes e Agostinho (1997) determinaram uma relação positiva entre o fator de condição de juvenis de curimba Prochilodus scrofa, da bacia do rio Paraná, e o seu recrutamento à pesca. Em seus resultados, os autores demonstraram que quando houve as maiores enchentes reguladas pela operaçáo da usina hidrelétrica de Itaipu, que possibilitaram maior expansão de habitats aquáticos e a maior oferta de alimento e abrigo, um maior fator de condição foi estimado para os juvenis de curimatã e, consequentemente, maior foi o sucesso de recrutamento pesqueiro e os índices de captura comercial desta espécie.

Neste sentido, um melhor aproveitamento dos recursos disponíveis em áreas de inundação de águas pretas pode ser um fator importante para a melhor condiçáo nutricional dos juvenis de $P$. nigricans nestes ambientes, pois o ganho em peso e comprimento dos juvenis nas áreas 2 e 3 não teve reflexo sobre um melhor status nutricional quando comparados aos juvenis de curimatã da área 1 . A alta seletividade alimentar de P. nigricans, como estabelecido por Yossa e Araújo-Lima (1998), pode dificultar a assimilação de grande quantidade de itens alimentares em áreas inundadas por águas brancas. Em ambientes inundados por águas pretas esta espécie encontraria maior facilidade para a seleção e assimilação dos itens alimentares, os quais são menos abundantes do que nas áreas de várzea. 
Além disso, Araújo-Lima et al. (1986) e Goulding et al. (1988) sugerem que os detritos de águas pretas, principalmente os ligados ao perifíton, apresentam um maior potencial de assimilaçáo para peixes detritívoros em virtude de sua alta qualidade protéica, indo ao encontro da hipótese de alta adaptação dos peixes detritívoros amazônicos aos sistemas aquáticos de águas pretas (Ribeiro e Petrere Jr. 1990).

\section{CONCLUSÕES}

Os resultados deste trabalho indicam que os juvenis de curimatá criados nas áreas de "igapó" apresentaram um melhor status nutricional do que os de águas brancas e, portanto, essas áreas podem apresentar um papel mais determinante no recrutamento e sustentabilidade da pesca de detritívoros nas proximidades de Manaus. Isto significa que uma das medidas mais parcimoniosas de gerenciamento pesqueiro para a manutenção dos estoques de peixes detritívoros da região está na conservação das áreas de inundação, em especial, aquelas sob influência do rio Negro.

\section{AGRADECIMENTOS}

Ao Dr. Jansen Zuanon, pela ajuda na identificação taxonômica do material. Aos Drs. Carlos Edwar de Carvalho Freitas e Alexandre Rivas pelo financiamento do estudo por meio do Projeto PIATAM IV (FINEP/PETROBRÁS). À M.Sc. Marina Bez pelo trabalho nas coletas e triagem do material.

\section{BIBLIOGRAFIA CITADA}

Araújo-Lima, C.A.R.M.; Forsberg, B.R.; Victoria, R.; Martinelli, L. 1986. Energy sources for detrivorous fishes in the Amazon. Science, 234: 1256-1258.

Araújo-Lima, C.A.R.M.; Oliveira, E.C. 1998. Transport of larval fish in the Amazon. Journal of Fish Biology, 53 (Suppl. A): 297-306.

Barthem, R.; Goulding, M. 2007. Un expected ecosystem: The Amazon as revealed by fisheries. Missouri Botanical Garden Press, Missouri (USA). 293 pp.

Batista, V.; Petrere Jr. M. 2003. Characterization of the commercial fish production landed at Manaus, Amazonas State, Brazil. Acta Amazonica, 33: 53-66.

Benedito-Cecílio, E.; Araújo-Lima, C.A.R.M.; Forsberg, B.R.; Bittencourt, M.M.; Martinelli, L.C. 2000. Carbon sources of Amazonian fisheries. Fisheries Management and Ecology, 7: 3015-315.

Benedito-Cecílio, E.; Araújo-Lima, C.A.R.M. 2002. Variation in the carbon isotope of Semaprochilodus insignis, a detritivorous fish associated with oligotrophic and eutrophic Amazon rivers. Journal of Fish Biology, 60: 1603-1607.

Forsberg, B.R.; Araújo-Lima, C.A.R.M.; Martinelli, L.A.; Victoria, R.L.; Bonassi, J.A. 1993. Autotrophic carbon sources for fish of the Central Amazon. Ecology, 74(3): 643-652.
Gomes, L.C.; Agostinho, A.A. 1997. Influence of the flooding regime on the nutritional state and juvenile recruitment of the curimba, Prochilodus scrofa, Steindachner, in upper Paraná River, Brazil. Fisheries Management and Ecology, 4: 263-274.

Goulding, M.; Carvalho, M.L.; Ferreira, E.G. 1988. Rio Negro, rich life in poor water. SPB Academic Publishing. The Hague, Netherlands. 200 pp.

Le Cren, E.D. 1951. The length-weight relationship and seasonal cycle in gonad weight and condition in the perch (Perca fuviatilis). Journal of Animal Ecology, 20(2): 201-219.

Leite, R.G.; Araújo-Lima, C.A.R.M. 2002. Feeding of the Brycon cephalus, Triportheus elongatus and Semaprochilodus insignis (OSTEICHTHYES, CHARACIFORMES) larvae in Solimóes/ Amazonas river and floodplain areas. Acta Amazonica, 32(3): 499-515.

Leite, R.G.; Araújo-Lima, C.A.R.M.; Victoria, R.L.; Martinelli, L.A. 2002. Stable isotope analysis of energy sources for larvae of eight fish species from the Amazon floodplain. Ecology of Freshwater Fish, 11: 56-63.

Lima, A.C.; Araújo-Lima, C.A.R.M. 2004. The distributions of larval and juvenile fishes in Amazonian rivers of different nutrient status. Freshwater Biology, 49(6): 787-800.

Petry, P.; Bayley, P.B.; Markle, D.F. 2003. Relationships between fish assemblages, macrophytes and environmental gradients in the Amazon River floodplain. Journal of Fish Biology, 63: 547-579.

Ribeiro, M.C.L.B.; Petrere Jr., M. 1990. Fisheries ecology and management of the jaraqui (Semaprochilodus Taeniurus, $S$. Insignis) in central Amazonia. 2006. Regulated Rivers: Research and Management, 5: 195-215.

Sanchéz-Botero, J.I.; Araújo-Lima, C.A.R.M. 2001. As macrófitas aquáticas como berçário para a ictiofauna da várzea do rio Amazonas. Acta Amazonica. 31(3): 437-447.

Santos, G.M.; Zuanon, J.A.S.; Ferreira, E.J. 2006. Peixes comerciais de Manaus. IBAMA/ProVarzea, Manaus (AM). 144 pp.

Silva-Soares, G. 2010. Fontes autotróficas de energia e dieta de Characiformes juvenis em herbáceas aquáticas do rio Solimôes/ Amazonas. Dissertação de mestrado, Instituto Nacional de Pesquisas da Amazônia, Manaus, Amazonas. 31 pp.

Sioli, H. 1984. The Amazon and its main affluents: hydrograph, morphology of the river courses, and river types, p.127-165. In: Sioli, H. (Ed.) The Amazon. W. Junk Publishers. Dordrecht, Holland.

Vazzoler, A.E.A.M. 1996. Biologia da reprodução de peixes teleósteos: teoria e prática. NUPELIA, Maringá (PR). 169 pp.

Yossa, M.I.; Araújo-Lima, C.A.R.M. 1998. Detritivory in two Amazonian fish species. Journal of Fish Biology, 52: 1141-1153.

Zar, J.H. 1996. Biostatistical Analysis. Prentice-Hall Inc. Englewood, England. 620 pp.

Recebido em 16/11/2011

Aceito em 28/07/2012 
